\title{
Partial dynamical symmetry and odd-even staggering in deformed nuclei
}

\author{
A. Leviatan ${ }^{1, a}$ \\ ${ }^{1}$ Racah Institute of Physics, The Hebrew University, Jerusalem 91904, Israel
}

\begin{abstract}
Partial dynamical symmetry (PDS) is shown to be relevant for describing the odd-even staggering in the $\gamma$-band of ${ }^{156} \mathrm{Gd}$ while retaining solvability and good $\mathrm{SU}(3)$ symmetry for the ground and $\beta$ bands. Several classes of interacting boson model Hamiltonians with SU(3) PDS are surveyed.
\end{abstract}

A convenient starting point for describing axiallydeformed nuclei is the SU(3) limit of the interacting boson model (IBM) [1]. The latter limit corresponds to the chain of nested algebras

$\begin{array}{ccccc}\mathrm{U}(6) & \supset & \mathrm{SU}(3) & \supset & \mathrm{SO}(3) \\ \downarrow & & \downarrow & & \downarrow \\ {[N]} & & (\lambda, \mu) & K & L\end{array}$

where below each algebra the associated labels of irreducible representations (irreps) are given, and $K$ is a multiplicity label. The eigenstates $|[N](\lambda, \mu) K, L\rangle$ are obtained with a Hamiltonian with SU(3) DS which has the form

$$
\hat{H}_{\mathrm{DS}}=\alpha_{1} \hat{C}_{2}[\mathrm{SU}(3)]+\alpha_{2} \hat{C}_{2}[\mathrm{SO}(3)]+\alpha_{3} \hat{C}_{3}[\mathrm{SU}(3)] \text {. }
$$

The quadratic and cubic Casimir operators of SU(3) are $\hat{C}_{2}[\operatorname{SU}(3)]=2 \hat{Q} \cdot \hat{Q}+\frac{3}{4} \hat{L} \cdot \hat{L}$ and $\hat{C}_{3}[\operatorname{SU}(3)]=-4 \sqrt{7} \hat{Q}$. $(\hat{Q} \times \hat{Q})^{(2)}-\frac{9}{2} \sqrt{3} \hat{Q} \cdot(\hat{L} \times \hat{L})^{(2)}$, and $\hat{C}_{2}[\mathrm{SO}(3)]=\hat{L} \cdot \hat{L}$. The SU(3) generators are $\hat{Q}=d^{\dagger} s+s^{\dagger} \tilde{d}-\frac{1}{2} \sqrt{7}\left(d^{\dagger} \tilde{d}\right)^{(2)}$ and $\hat{L}=$ $\sqrt{10}\left(d^{\dagger} \tilde{d}\right)^{(1)}$. The monopole $(s)$ and quadrupole $(d)$ bosons represent valence nucleon pairs whose total number $N$ is conserved. $\hat{H}_{D S}$ is completely solvable with eigenenergies

$$
E_{\mathrm{DS}}=\alpha_{1} f_{2}(\lambda, \mu)+\alpha_{2} L(L+1)+\alpha_{3} f_{3}(\lambda, \mu),
$$

where $f_{2}(\lambda, \mu)=\lambda^{2}+(\lambda+\mu)(\mu+3)$ and $f_{3}(\lambda, \mu)=(\lambda-\mu)(2 \lambda+\mu+$ $3)(\lambda+2 \mu+3)$. The spectrum resembles that of a quadrupole axially-deformed rotor with eigenstates arranged in $\mathrm{SU}(3)$ multiplets and $K$ corresponds geometrically to the projection of the angular momentum on the symmetry axis. The lowest SU(3) irrep $(2 N, 0)$ contains the ground band $\mathrm{g}(K=0)$, while $(2 N-4,2)$ encompasses the $\beta(K=0)$ and $\gamma(K=2)$ bands. The in-band rotational spectrum is that of a rigid rotor with characteristic $L(L+1)$ splitting for all $K$-bands.

A comparison with the experimental spectrum and E2 rates of ${ }^{156} \mathrm{Gd}$ is shown in Figs. 1-2 and Table 1. It displays a good description for properties of states in the ground and $\beta$ bands. However, the resulting fit to energies of the

\footnotetext{
ae-mail: ami@phys.huji.ac.il
}

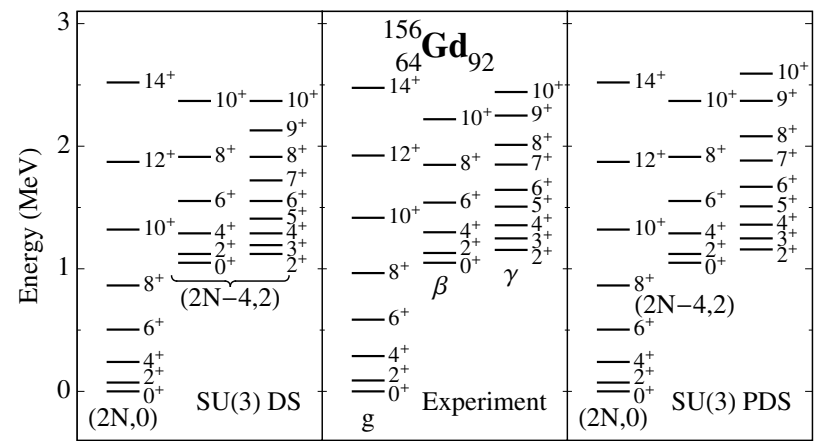

Figure 1. Observed spectrum of ${ }^{156} \mathrm{Gd}$ [2] compared with the calculated spectra of $\hat{H}_{\mathrm{DS}}$ (2) with SU(3) DS and of $\hat{H}_{\mathrm{PDS}}$ (9) with SU(3) PDS for $N=12$. The parameters are $\alpha_{1}=-7.6, \alpha_{2}=$ 12.0, $\alpha_{3}=0 \mathrm{keV}$ in both Hamiltonians and $\eta_{1}=-0.23, \eta_{3}=$ $1.54 \mathrm{keV}$ in $\hat{H}_{\mathrm{PDS}} .(\lambda, \mu)$ are $\mathrm{SU}(3)$ labels. Adapted from [13].

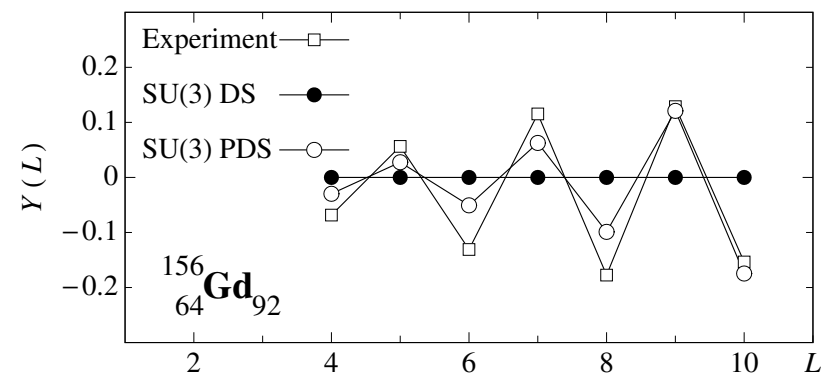

Figure 2. Observed [2] and calculated (SU(3) DS and PDS) oddeven staggering of the $\gamma$ band in ${ }^{156} \mathrm{Gd}$. Adapted from [13].

$\gamma$-band is quite poor. The latter display an odd-even staggering with pronounced deviations from a rigid-rotor pattern, indicative of a triaxial behavior. This effect can be visualized by plotting the quantity [3]

$$
Y(L)=\frac{2 L-1}{L} \times \frac{E(L)-E(L-1)}{E(L)-E(L-2)}-1
$$


Table 1. Observed [2] and calculated $B(\mathrm{E} 2)$ values in ${ }^{156} \mathrm{Gd}$. For both the DS and PDS calculations, the E2 operator is $e_{\mathrm{b}}\left[s^{\dagger} \tilde{d}+d^{\dagger} s+\chi\left(d^{\dagger} \tilde{d}\right)^{(2)}\right]$ with an effective boson charge $e_{\mathrm{b}}=0.166 e b$ and $\chi=-0.168$. Adapted from [13].

\begin{tabular}{llll|llll}
\hline Transition & Experiment & DS & PDS & Transition & Experiment & DS & PDS \\
\hline $2_{1}^{+} \rightarrow 0_{1}^{+}$ & 0.93325 & 0.933 & 0.933 & $4_{\beta}^{+} \rightarrow 2_{1}^{+}$ & 0.006535 & 0.0067 & 0.0067 \\
$4_{1}^{+} \rightarrow 2_{1}^{+}$ & 1.31225 & 1.313 & 1.313 & $4_{\beta}^{+} \rightarrow 4_{1}^{+}$ & - & 0.0067 & 0.0067 \\
$6_{1}^{+} \rightarrow 4_{1}^{+}$ & 1.47240 & 1.405 & 1.405 & $4_{\beta}^{+} \rightarrow 6_{1}^{+}$ & 0.010555 & 0.021 & 0.021 \\
$8_{1}^{+} \rightarrow 6_{1}^{+}$ & 1.59685 & 1.409 & 1.409 & $2_{\gamma}^{+} \rightarrow 0_{1}^{+}$ & 0.02338 & 0.035 & 0.030 \\
$10_{1}^{+} \rightarrow 8_{1}^{+}$ & 1.56670 & 1.364 & 1.364 & $2_{\gamma}^{+} \rightarrow 2_{1}^{+}$ & 0.036112 & 0.056 & 0.048 \\
$2_{\beta}^{+} \rightarrow 0_{\beta}^{+}$ & 0.2611 & 0.679 & 0.679 & $2_{\gamma}^{+} \rightarrow 4_{1}^{+}$ & 0.00382 & 0.0037 & 0.0031 \\
$4_{\beta}^{+} \rightarrow 2_{\beta}^{+}$ & 1.4075 & 0.951 & 0.951 & $3_{\gamma}^{+} \rightarrow 2_{1}^{+}$ & 0.036470 & 0.062 & 0.053 \\
$0_{\beta}^{+} \rightarrow 2_{1}^{+}$ & 0.042 & 0.034 & 0.034 & $3_{\gamma}^{+} \rightarrow 4_{1}^{+}$ & 0.025450 & 0.032 & 0.028 \\
$2_{\beta}^{+} \rightarrow 0_{1}^{+}$ & 0.00313 & 0.0055 & 0.0055 & $4_{\gamma}^{+} \rightarrow 2_{1}^{+}$ & 0.009025 & 0.017 & 0.015 \\
$2_{\beta}^{+} \rightarrow 2_{1}^{+}$ & 0.016515 & 0.0084 & 0.0084 & $4_{\gamma}^{+} \rightarrow 4_{1}^{+}$ & 0.05015 & 0.067 & 0.057 \\
$2_{\beta}^{+} \rightarrow 4_{1}^{+}$ & 0.020420 & 0.020 & 0.020 & $4_{\gamma}^{+} \rightarrow 6_{1}^{+}$ & - & 0.0089 & 0.0076 \\
& & & & $4_{\gamma}^{+} \rightarrow 2_{\beta}^{+}$ & 0.021480 & 0.0033 & 0.0096 \\
\hline
\end{tabular}

where $E(L)$ is the excitation energy of a $\gamma$-band level with angular momentum $L$. For a rotor this quantity is flat, $Y(L)=0$, as illustrated in Fig. 2 with the SU(3) DS calculation, in marked disagreement with the empirical data. A proper treatment of such signature splitting necessitates the inclusion of at least cubic terms in the IBM Hamiltonian. In the IBM there are 17 possible three-body interactions. One is thus confronted with the need to select suitable higher-order terms that can break the DS in the $\gamma$-band but preserve it in the ground and $\beta$ bands. These are precisely the defining properties of a partial dynamical symmetry (PDS) [4]. The essential idea is to relax the stringent conditions of complete solvability, so that only part of the eigenspectrum retains all the DS quantum numbers. Various types of bosonic and fermionic PDS are known to be relevant to nuclear spectroscopy [4-13]. In the present contribution we demonstrate the relevance of PDS to the odd-even staggering in the $\gamma$-band of ${ }^{156} \mathrm{Gd}$ [13].

The method to construct Hamiltonians with PDS is based on an expansion in terms of tensors which annihilate prescribed set of states $[12,14]$

$$
\hat{H}_{\mathrm{PDS}}=\sum_{\alpha, \beta} u_{\alpha \beta} \hat{B}_{\alpha}^{\dagger} \hat{B}_{\beta} .
$$

In the present study, the tensors involve $n$-boson creation and annihilation operators $\hat{B}_{\alpha}^{\dagger} \equiv \hat{B}_{[n](\lambda, \mu) K ; \ell m}^{\dagger}$ and $\hat{B}_{\alpha}$, with definite character under the $\mathrm{SU}(3)$ chain (1), which satisfy

$$
\hat{B}_{\alpha}|[N](2 N, 0), K=0, L\rangle=0
$$

Interactions as in Eq. (5) can be added to the Hamiltonian (2) without destroying solvability of part of its spectrum. For $n=2$, we find two such SU(3) tensors

$$
\begin{array}{lll}
\hat{B}_{[2](0,2) 0 ; 00}^{\dagger} & \propto & P_{0}^{\dagger}=d^{\dagger} \cdot d^{\dagger}-2\left(s^{\dagger}\right)^{2}, \\
\hat{B}_{[2](0,2) 0 ; 2 m}^{\dagger} & \propto & P_{2 m}^{\dagger}=2 d_{m}^{\dagger} s^{\dagger}+\sqrt{7}\left(d^{\dagger} d^{\dagger}\right)_{m}^{(2)},
\end{array}
$$

with $(\lambda, \mu)=(0,2)$ and angular momentum $\ell=0,2$. For $n=3$, we find six $\operatorname{SU}(3)$ tensors

$$
\begin{array}{lll}
\hat{B}_{[3](2,2) 0 ; 00}^{\dagger} & \propto & W_{0}^{\dagger}=5 P_{0}^{\dagger} s^{\dagger}-P_{2}^{\dagger} \cdot d^{\dagger}, \\
\hat{B}_{[3](2,2) 2 ; 2 m}^{\dagger} & \propto & W_{2 m}^{\dagger}=P_{0}^{\dagger} d_{m}^{\dagger}+2 P_{2 m}^{\dagger} s^{\dagger}, \\
\hat{B}_{[3](2,2) 0 ; 2 m}^{\dagger} & \propto & V_{2 m}^{\dagger}=6 P_{0}^{\dagger} d_{m}^{\dagger}-P_{2 m}^{\dagger} s^{\dagger}, \\
\hat{B}_{[3](2,2) 2 ; \ell m}^{\dagger} & \propto & W_{\ell m}^{\dagger}=\left(P_{2}^{\dagger} d^{\dagger}\right)_{m}^{(\ell)} \quad \ell=3,4, \\
\hat{B}_{[3](0,0) 0 ; 00}^{\dagger} & \propto & \Lambda^{\dagger}=P_{0}^{\dagger} s^{\dagger}+P_{2}^{\dagger} \cdot d^{\dagger},
\end{array}
$$

with $(\lambda, \mu)=(2,2), \ell=0,2,2,3,4$ and $(\lambda, \mu)=(0,0)$, $\ell=0$, all satisfying Eq. (6)

The aforementioned Casimir operators of SU(3) and the integrity basis operator $\hat{\Omega}=-4 \sqrt{3} \hat{Q} \cdot(\hat{L} \times \hat{L})^{(2)}$, which conserve $\mathrm{SU}(3)$, are contained in the expression (5). In general, however, $\hat{H}_{\mathrm{PDS}}(5)$ breaks SU(3) yet, by construction, for any choice of parameters the ground-band members $\|[N](2 N, 0) K=0, L\rangle$ are solvable with good $\mathrm{SU}(3)$ symmetry. For specific choices, additional solvable states are obtained, as we outline below.

Of particular interest is a class of Hamiltonians with SU(3) PDS which has solvable ground and $\beta$ bands with good SU(3) symmetry and energies $E_{\mathrm{DS}}(3)$. This follows from the structure of the relevant Hamiltonian,

$$
\hat{H}_{\mathrm{PDS}}=\hat{H}_{\mathrm{DS}}+\eta_{2} W_{2}^{\dagger} \cdot \tilde{W}_{2}+\eta_{3} W_{3}^{\dagger} \cdot \tilde{W}_{3},
$$

and the fact that $W_{2 m}(8 \mathrm{~b})$ and $W_{3 m}(8 \mathrm{~d})$ satisfy Eq. (6) and

$$
W_{\ell m}|[N](2 N-4,2), K=0, L\rangle=0 \quad \ell=2,3 .
$$

The eigenstates of these solvable bands, $\mid[N](2 N, 0) K=$ $0, L\rangle$ and $|[N](2 N-4,2,0) K=0, L\rangle$, are the same for the DS and PDS Hamiltonians. In contrast, other bands including the $\gamma$ band are mixed with respect to $\mathrm{SU}(3)$. As shown in Fig. 2, the empirical odd-even staggering in the $\gamma$-band in ${ }^{156} \mathrm{Gd}$ is well reproduced by the PDS Hamiltonian (9). The calculated staggering increases with $L$ which agrees with the experiment up to $L=10$. For the PDS calculation, the wave functions of the states in the $\gamma$ band involve $15 \% \mathrm{SU}(3)$ admixtures into the dominant $(2 N-4,2)$ component. These admixtures reflect the coupling of the 


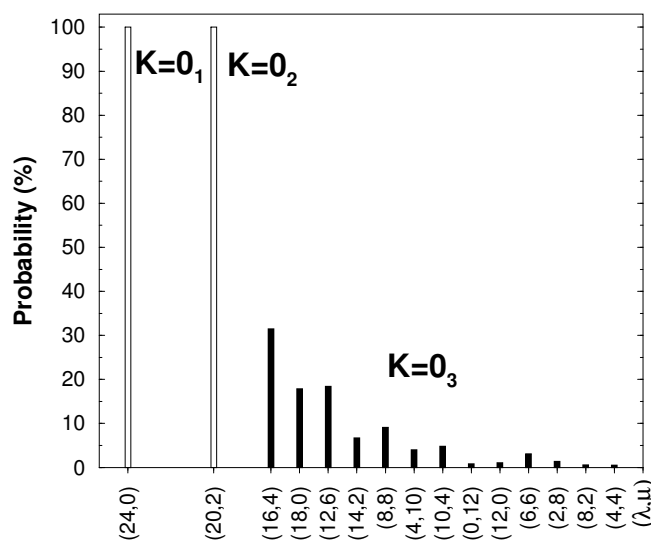

Figure 3. SU(3) decomposition of wave functions of $L=0$ states in the $K=0_{1}, 0_{2}, 0_{3}$ bands for the PDS calculation. Adapted from [13].

$\gamma$ band with higher-lying excited bands. Other approaches advocating the coupling of the $\gamma$ band to the $\beta$ band [15] or to the ground band [16] fail to describe the odd-even staggering in ${ }^{156} \mathrm{Gd}$. Higher bands exhibit larger SU(3) mixing and their wave functions are spread over many $\mathrm{SU}(3)$ irreps, as shown for the $K=0_{3}$ band in Fig. 3. This complex $\mathrm{SU}(3)$ decomposition is in marked contrast to the $\mathrm{SU}(3)$ purity of the ground $\left(K=0_{1}\right)$ and $\beta\left(K=0_{2}\right)$ bands.

It should be emphasized that the PDS results for the $\gamma$ band are obtained without altering the good agreement for the ground and $\beta$ bands, already achieved with the SU(3) DS calculation. This is further illustrated with the E2 transitions in ${ }^{156} \mathrm{Gd}$. The observed $B(\mathrm{E} 2)$ values between ground, $\beta$, and $\gamma$ bands are shown in Table 1 and compared to the results of the SU(3) DS and PDS calculations. The $\mathrm{E} 2$ transitions between ground and $\beta$ bands can be calculated analytically, and remain valid in SU(3) PDS. Transitions involving $\gamma$-band members are different in SU(3) DS and PDS, and are computed numerically for the latter. It is seen from Table 1 that the mixing of the $\gamma$ band with higher-lying excited bands improves the agreement with the data in most cases.

Another class of Hamiltonians with SU(3) PDS exists which has solvable members of $\gamma^{k}(K=2 k)$ bands $|[N](2 N-4 k, 2 k) K=2 k, L\rangle$, with energies $E_{\mathrm{DS}}$ (3). This follows from the structure of the relevant Hamiltonian,

$$
\begin{aligned}
\hat{H}_{\mathrm{PDS}}^{\prime}= & \hat{H}_{D S}+t_{0} P_{0}^{\dagger} P_{0}+u_{0} P_{0}^{\dagger} s^{\dagger} s P_{0} \\
& +v_{0}\left(\Lambda^{\dagger} s P_{0}+P_{0}^{\dagger} s^{\dagger} \Lambda\right),
\end{aligned}
$$

and the fact that $P_{0}$ (7a) and $\Lambda$ (8e) satisfy Eq. (6) and

$$
\begin{aligned}
& P_{0}|[N](2 N-4 k, 2 k) K=2 k, L\rangle=0, \\
& \Lambda|[N](2 N-4 k, 2 k) K, L\rangle=0 .
\end{aligned}
$$

Two-body Hamiltonians of this class have been shown to play a role in diverse phenomena, including spectroscopy of rare-earth nuclei [5-7], quantum phase transitions [17, $18]$ and mixed regular and chaotic dynamics $[18,19]$. The two classes of SU(3)-PDS Hamiltonians demonstrate the increase in flexibility obtained by generalizing the concept of DS to PDS. In fact, in the IBM more than half of all possible interactions have an SU(3) PDS [13].

In summary, we have presented several classes of IBM Hamiltonians with SU(3) PDS, and obtained an improved description of signature splitting in the $\gamma$ band of ${ }^{156} \mathrm{Gd}$. The analysis serves to highlight the merits gained by using the notion of PDS as a tool for selecting higher-order terms in systems where a prescribed symmetry is not obeyed uniformly. On one hand, the PDS approach allows more flexibility by relaxing the constraints of an exact DS. On the other hand, the PDS picks particular symmetry-breaking terms without destroying results previously obtained with a DS for a segment of the spectrum. The non-solvable states can experience strong symmetry-breaking. These virtues can be exploited in studying the role of higherorder terms in collective Hamiltonians and in attempts to extend beyond-mean-field methods to heavy nuclei.

This work was done in collaboration with J.E. GarcíaRamos (Huelva) and P. Van Isacker (GANIL) and is supported by the Israel Science Foundation.

\section{References}

[1] F. Iachello and A. Arima, The Interacting Boson Model (Cambridge Univ. Press, Cambridge, 1987)

[2] C.W. Reich, Nucl. Data Sheets 99, 753 (2003)

[3] R.F. Casten, N.V. Zamfir, P. von Brentano, F. Seiffert and W. Lieberz, Phys. Lett. B 265, 9 (1991)

[4] A. Leviatan, Prog. Part. Nucl. Phys. 66, 93 (2011)

[5] A. Leviatan, Phys. Rev. Lett. 77, 818 (1996)

[6] A. Leviatan and I. Sinai, Phys. Rev. C 60, 061301 (1999)

[7] R.F. Casten, R.B. Cakirli, K. Blaum, and A. Couture, Phys. Rev. Lett. 113, 112501 (2014)

[8] A. Leviatan and P. Van Isacker, Phys. Rev. Lett. 89, 222501 (2002)

[9] C. Kremer, J. Beller, A. Leviatan, N. Pietralla, G. Rainovski, R. Trippel, and P. Van Isacker, Phys. Rev. C 89, 041302(R) (2014)

[10] J. Escher and A. Leviatan, Phys. Rev. Lett. 84, 1866 (2000); Phys. Rev. C 65, 054309 (2002)

[11] P. Van Isacker and S. Heinze, Phys. Rev. Lett. 100, 052501 (2008); Ann. Phys. 349, 73 (2014)

[12] J.E. García-Ramos, A. Leviatan and P. Van Isacker, Phys. Rev. Lett. 102, 112502 (2009)

[13] A. Leviatan, J.E. García-Ramos and P. Van Isacker, Phys. Rev. C 87, 021302(R) (2013)

[14] Y. Alhassid and A. Leviatan, J. Phys. A 25, L1265 (1992)

[15] D. Bonatsos, Phys. Lett. B 200, 1 (1988)

[16] N. Minkov, S.B. Drenska, P.P. Raychev, R.P. Roussev and D. Bonatsos, Phys. Rev. C 61, 064301 (2000)

[17] A. Leviatan, Phys. Rev. Lett. 98, 242502 (2007)

[18] M. Macek and A. Leviatan, Ann. Phys. 351, 302 (2014)

[19] N. Whelan, Y. Alhassid and A. Leviatan, Phys. Rev. Lett. 71, 2208 (1993) 
